\title{
Optimal Power Control for Rayleigh-Faded Multiuser Systems With Outage Constraints
}

\author{
John Papandriopoulos, Student Member, IEEE, Jamie Evans, Member, IEEE, and Subhrakanti Dey, Member, IEEE
}

\begin{abstract}
How can we achieve the conflicting goals of reduced transmission power and increased capacity in a wireless network, without attempting to follow the instantaneous state of a fading channel? In this paper, we address this problem by jointly considering power control and multiuser detection (MUD) with outage-probability constraints in a Rayleigh fast-fading environment. The resulting power-control algorithms (PCAs) utilize the statistics of the channel and operate on a much slower timescale than traditional schemes. We propose an optimal iterative solution that is conceptually simple and finds the minimum sum power of all users while meeting their outage targets. Using a derived bound on outage probability, we introduce a mapping from outage to average signal-to-interference ratio (SIR) constraints. This allows us to propose a suboptimal iterative scheme that is a variation of an existing solution to a joint power control and MUD problem involving SIR constraints. We further use a recent result that transforms complex SIR expressions into a compact and decoupled form, to develop a noniterative and computationally inexpensive PCA for large systems of users. Simulation results are presented showing the closeness of the optimal and mapped schemes, speed of convergence, and performance comparisons.
\end{abstract}

Index Terms-Code division multiple access (CDMA), large system analysis (LSA), multiuser detection (MUD), outage probability, power control, Rayleigh fading.

\section{INTRODUCTION}

$\mathbf{P}$ OWER control of transmitters in a wireless network is an effective way to improve performance and utilization, whether it be in an interference-limited system such as code division multiple access (CDMA), or a multiple-access system employing frequency reuse among cells as in frequency division multiple access (FDMA). The problem of allocating powers in a meaningful way such that users need only expend sufficient power to meet their quality-of-service (QoS) specifications has been studied extensively. Early work involved solving an eigenvalue problem for nonnegative matrices, where an approach known as signal-to-interference ratio (SIR) balancing was used to maximize the minimum SIR of the system

Manuscript received May 8, 2003; revised February 20, 2004; accepted October 18, 2004. The editor coordinating the review of this paper and approving it for publication is Q. Zhang. This paper was supported by the Australian Research Council.

The authors are with the Australian Research Council (ARC) Special Research Center for Ultra-Broadband Information Networks (CUBIN), Department of Electrical and Electronic Engineering, University of Melbourne, Melbourne VIC 3010, Australia. CUBIN is an affiliated program of National ICT Australia (NICTA) (e-mail: jpap@ee.unimelb.edu.au; jse@ee.unimelb. edu.au; sdey@ee.unimelb.edu.au).

Digital Object Identifier 10.1109/TWC.2005.858019
[1]-[4]. The optimal solution was found to occur when all SIRs are equal, or balanced, and was given by the eigenvector associated with the maximum eigenvalue of a nondegenerate channel-gain matrix.

Iterative and distributed power-control algorithms (PCAs) that converge each user's power to the minimum power result [5]-[9] have also been studied and are of practical importance: They utilize only local measurements to yield a global result close or equal to the optimal. In contrast, centralized algorithms require global knowledge of parameters; in practice, this translates into a requirement for low latency and high-bandwidth communication paths between base-station receivers (BSRs) in a multicellular network. A survey of this early work is given in [10]. More recently, convex optimization-based approaches have been investigated with the aim of finding the globally optimal result through centralized interior-point and primaldual methods [11], [12].

Much of this prior work considered quasi-stationary channel models; thus, to cope with a dynamic fading environment, solutions to the respective problems would need to be reevaluated each time the environment changed. This implies that such methods would need to be highly efficient to be implemented in practice, or incur a digital signal processing power penalty due to intense signal processing requirements. Such methods may also require frequent communication between mobile stations and their assigned base station at each reevaluation. Work capturing the dynamics of the channel include those in [13] and [14], where channel gains are not assumed to be known exactly; noise-corrupted estimates are used instead, resulting in stochastic PCAs.

In this paper, we consider a dynamic fading environment and a power-control scheme that does not attempt to follow the instantaneous fading state of the channel. We simply relax the traditional SIR constraints by allowing the received power of each user to drop below the prescribed threshold with low probability. Such a power-control problem with outageprobability constraints operates on a slower timescale, following lognormal shadowing variations for instance, and was first considered in [12]. It has many advantages: for example, should the fading severity and duration increase, we can more naturally cater for delay-constrained situations by declaring outage rather than the increasing the interleaving depth in vain. A consequence of the less frequent power updates, we are also able to avoid the efficiency problems identified above, including situations where power warfare may result from iterative schemes that cannot keep up with the instantaneous fading state of the channel. Ultimately however, the tradeoff with such an 
outage-based scheme is an increased power allocation to give an extra margin of SIR. In return, the probability of fast-fadinginduced outage is reduced to an acceptable level that attains the given QoS specifications.

The original problem considered in [12] was solved by a nonlinear optimization, utilizing interior-point methods for numerical implementation, while receiver noise was neglected in the interest of convexity. In this paper, we show how a similar problem that includes receiver noise can be solved using a conceptually simple iterative algorithm fitting within the framework given in [15]. We extend and enhance the problem to jointly include multiuser detection (MUD). It is well known that in a multiple-access system, MUD can be used to further enhance the performance of wireless systems by exploiting the structure of the multiple-access interference [16]. We introduce a new minimum outage-probability (MOP) multiuser receiver that finds a linear receiver filter to minimize outage. Should this receiver be allowed to directly depend on the fast-fading coefficients, this would be nothing more than the well-known linear minimum mean squared error (MMSE) receiver [17], [18] since it is SIR maximizing. However, in this paper, both power control and receiver updates are specifically designed to be independent of the fast-fading coefficients. Through a joint optimization of both power and linear receivers, an increase in user capacity in addition to a reduction of power consumption is possible [19].

Traditionally, power allocation and MUD were considered separately: power allocation assumed a fixed receiver structure and MUD assumed fixed user transmit powers. Only recently has such a joint optimization of both user transmit powers and receiver filters been considered [20]-[22]. However, all literature to date deals with a joint optimization having SIR constraints-there is a lack of work dealing with a joint optimization to minimize outage probability. In this paper, we propose three novel iterative methods to solve such a problem: An optimal method that utilizes the MOP multiuser receiver, a suboptimal method that utilizes the MMSE receiver, and another method that is a hybrid of the former two.

We investigate bounds on outage probability in a Rayleigh fading environment, showing that with receiver noise, these bounds have the same form and tightness as the noiseless case in [12]. Using the upper bound, we derive a relationship between outage probability and average SIR, allowing a mapping to take place between outage and average SIR constraints. Now, only considering these average SIR constraints, we can leverage prior work dealing with instantaneous SIR constraints, except we now consider the problem on a slower time scale. We illustrate this idea by considering a large system analysis (LSA) of a wireless network. Traditionally, a major difficulty in the analysis of a power-controlled environment is the coupling of all users through the interference they cause each other. One simplifying case is the decorrelating detector; however, recent work has shown that in a large system, decoupling of the interfering effects is also possible for several other receiver structures including the MMSE receiver [19]. We use results from this paper to create a decentralized power-control scheme that requires little computation and yields good results for finite-sized systems.
To summarize, the main contributions of this paper are the following.

1) The design of a conceptually simple iterative algorithm that minimizes the sum power of all users subject to outage-probability constraints. The optimization is performed on choice of user powers and linear multiuser receivers. This problem reduces to that considered in [12] if background noise is neglected and linear receivers fixed.

2) A novel suboptimal iterative PCA and MUD (PCAMUD) where outage constraints are first mapped to average SIR threshold constraints. Such a mapping permits the use of a variant of an existing PCA-MUD given in [20], which is computationally efficient.

3) A further suboptimal iterative PCA-MUD that combines the concepts of the above two, yielding results closer to the optimal, yet with a similar computational efficiency to the previous algorithm.

4) A decentralized noniterative PCA-MUD for medium to large systems of users using a recent result that transforms complex expressions for SIR into a compact and decoupled form.

The paper is organized as follows. In Section II, we introduce our system and fading model. Section III derives expressions for SIR, user outage probability, and new upper and lower bounds relating to a margin of average SIR, when all statistical variations in signal and noise power are ignored. In Section IV, we formulate a joint power-control and multiuser-receiver optimization problem and give an iterative algorithm that yields the optimal solution. Section V derives a mapping between outage probability and average SIR, then formulates a suboptimal joint power-control and multiuser-receiver optimization problem based on such a mapping. Section VI introduces a hybrid PCA-MUD that combines the concepts of the optimal PCA with the MMSE multiuser receiver. In Section VII, largesystem results are derived. Section VIII provides simulation results showing the closeness of all iterative schemes and a comparison to the noniterative PCA-MUD for large systems. Finally, Section IX contains our conclusions and outlines some open issues for further investigation.

\section{SYSTEM MODEL}

In this paper, we consider the uplink in a direct-sequence synchronous CDMA (DS-SCDMA) communications system with $K$ users and a processing gain of $N$. We assume a BPSK modulation scheme and an $N$-dimensional chip matched-filter (MF) vector at each receiver $i$. The received signal at each filter input is given by

$$
\mathbf{r}_{i}=\sum_{j=1}^{K} \sqrt{G_{i j} F_{i j} P_{j}} b_{j} \mathbf{s}_{j}+\mathbf{n}_{i}, \quad i=1, \ldots, K
$$

where

- $G_{i j}$ is the positive slow-varying path gain of user $j$ to the assigned base station of user $i$,

- $F_{i j}$ is the associated fast-fading component of the channel, 
- $P_{j}$ is the transmit power of user $j$,

- $b_{j}$ are data bits taking on values of \pm 1 with equal probability,

- $\mathbf{s}_{j}$ is the fixed $N$-dimensional spreading sequence of user $j$ with elements taking values $\pm 1 / \sqrt{N}$,

- $\mathbf{n}_{i}$ is assumed to be additive white Gaussian noise (AWGN) with zero mean and covariance $\sigma^{2} \mathbf{I}$,

and we further define $\mathbf{S}=\left[\mathbf{s}_{1}, \ldots, \mathbf{s}_{K}\right]$.

More specifically, the terms $F_{i j}$ model fast time scale Rayleigh fading and are assumed to be unit-mean exponentially distributed random variables, independent for all $j$ given $i$. In this so-called Rayleigh/Rayleigh fading environment, the received power has mean value

$$
E\left[G_{i j} F_{i j} P_{j}\right]=G_{i j} P_{j} .
$$

The analysis that follows holds only over a time scale where factors affecting $G_{i j}$ do not change significantly.

Let $\mathbf{c}_{i}$ denote the linear receiver for user $i$ at its assigned BSR and $\mathbf{C}=\left[\mathbf{c}_{1}, \ldots, \mathbf{c}_{K}\right]$. The filter output of user $i$ at its assigned $\mathrm{BSR}$ is given by

$$
\mathbf{y}_{i}=\mathbf{c}_{i}^{\mathrm{T}} \mathbf{r}_{i}=\sum_{j=1}^{K} \sqrt{G_{i j} F_{i j} P_{j}}\left(\mathbf{c}_{i}^{\mathrm{T}} \mathbf{s}_{j}\right) b_{j}+\tilde{n}_{i}
$$

where $\tilde{n}_{i}=\mathbf{c}_{i}^{\mathrm{T}} \mathbf{n}_{i}$ is $N\left(0, \sigma^{2} \mathbf{c}_{i}^{\mathrm{T}} \mathbf{c}_{i}\right)$.

\section{OUtage PRobability AND CeRtainty-Equivalent MARGIN (CEM) WITH NOISE}

To simplify the notation in this section, we shall drop the receiver filter terms $\left(\mathbf{c}_{i}^{\mathrm{T}} \mathbf{s}_{j}\right)^{2}$ without loss of generality (since we can absorb them into the $G_{i j}$ terms). They will become important in Section IV-B.

\section{A. SIR and Outage Probability}

The $\operatorname{SIR} \gamma$ of user $i$ is given by

$$
\gamma_{i}=\frac{G_{i i} F_{i i} P_{i}}{\sum_{j \neq i} G_{i j} F_{i j} P_{j}+\sigma^{2}} .
$$

The outage probability of user $i$, denoted $O_{i}$, is defined as the proportion of time that some SIR threshold $\gamma_{i}^{\text {th }}$ is not met for sufficient reception at the BSR. By a careful choice of $\gamma_{i}^{\text {th }}$, we can set the QoS for each user. We can express the outage probability for the $i$ th user as

$$
\begin{aligned}
O_{i} & =\operatorname{Pr}\left(\gamma_{i} \leq \gamma_{i}^{\mathrm{th}}\right) \\
& =\operatorname{Pr}\left(G_{i i} F_{i i} P_{i} \leq \gamma_{i}^{\mathrm{th}}\left\{\sum_{j \neq i} G_{i j} F_{i j} P_{j}+\sigma^{2}\right\}\right) .
\end{aligned}
$$

In a Rayleigh/Rayleigh fading environment, we have (see [12])

$$
O_{i}=1-\exp \left(\frac{-\sigma^{2} \gamma_{i}^{\mathrm{th}}}{G_{i i} P_{i}}\right) \prod_{j \neq i} \frac{1}{1+\frac{\gamma_{i}^{\mathrm{th}} G_{i j} P_{j}}{G_{i i} P_{i}}} .
$$

\section{B. CEM With Noise}

The CEM was defined in [12] without noise. It represents a margin of error for average SIR when representing the system by a certainty-equivalent form (with all statistical variation in signal and noise power ignored and replaced with their expected values).

We will take the average SIR (denoted $\overline{S I R}$ ) to mean the expected value of the $i$ th user's received power over the expected value of the interference from the $K-1$ other users and background noise. This is also the certainty-equivalent SIR and is given by

$$
\overline{S I R}_{i}=\frac{E\left[G_{i i} F_{i i} P_{i}\right]}{E\left[\sum_{j \neq i} G_{i j} F_{i j} P_{j}+\sigma^{2}\right]}=\frac{G_{i i} P_{i}}{\sum_{j \neq i} G_{i j} P_{j}+\sigma^{2}} .
$$

As with [12], we also define the CEM (with noise, denoted $\mathrm{CEM}^{\sigma}$ ) as the ratio of the certainty-equivalent $\overline{\mathrm{SIR}}$ to the average SIR threshold

$$
C E M_{i}^{\sigma}=\frac{\overline{S I R}_{i}}{\gamma_{i}^{\text {th }}}=\frac{G_{i i} P_{i}}{\gamma_{i}^{\text {th }}\left\{\sum_{j \neq i} G_{i j} P_{j}+\sigma^{2}\right\}} .
$$

\section{Relation Between the $C E M^{\sigma}$ and Outage Probability}

We make use of the general result

$$
1+k+\sum_{i}^{n} z_{i} \leq \mathrm{e}^{k} \prod_{i}^{n}\left(1+z_{i}\right) \leq \mathrm{e}^{k+\sum_{i}^{n} z_{i}}
$$

which is valid for any $z_{1}, \ldots, z_{n}, k \geq 0$ and can easily be proved by following [12, Appendix II]. We shall use this result to find the upper and lower bounds on outage probability in terms of the $\mathrm{CEM}^{\sigma}$.

From (5), we have

$$
O_{i}=1-\left[\exp \left(\frac{\sigma^{2} \gamma_{i}^{\mathrm{th}}}{G_{i i} P_{i}}\right) \prod_{j \neq i}\left(1+\frac{\gamma_{i}^{\mathrm{th}} G_{i j} P_{j}}{G_{i i} P_{i}}\right)\right]^{-1} .
$$

Using the left-hand side inequality in (8)

$$
\begin{aligned}
O_{i} & \geq 1-\left[1+\frac{\sigma^{2} \gamma_{i}^{\text {th }}}{G_{i i} P_{i}}+\sum_{j \neq i} \frac{\gamma_{i}^{\text {th }} G_{i j} P_{j}}{G_{i i} P_{i}}\right]^{-1} \\
& =1-\left[1+\frac{\gamma_{i}^{\text {th }}\left\{\sum_{j \neq i} G_{i j} P_{j}+\sigma^{2}\right\}}{G_{i i} P_{i}}\right]^{-1} \\
& =1-\frac{1}{1+\frac{1}{C E M_{i}^{\sigma}}} \\
& =\frac{1}{1+C E M_{i}^{\sigma}} .
\end{aligned}
$$


Repeating the procedure above for the right-hand side inequality in (8)

$$
\begin{aligned}
O_{i} & \leq 1-\exp \left(-\frac{\sigma^{2} \gamma_{i}^{\mathrm{th}}}{G_{i i} P_{i}}-\sum_{j \neq i} \frac{\gamma_{i}^{\mathrm{th}} G_{i j} P_{j}}{G_{i i} P_{i}}\right) \\
& =1-\exp \left(-\frac{\gamma_{i}^{\mathrm{th}}\left\{\sum_{j \neq i} G_{i j} P_{j}+\sigma^{2}\right\}}{G_{i i} P_{i}}\right) \\
& =1-\exp \left(-\frac{1}{C E M_{i}^{\sigma}}\right) .
\end{aligned}
$$

The upper and lower bounds on outage are thus

$$
\frac{1}{1+C E M_{i}^{\sigma}} \leq O_{i} \leq 1-\exp \left(-\frac{1}{C E M_{i}^{\sigma}}\right)
$$

which have the same form and tightness in the region of interest as the noiseless case in [12].

\section{Optimal Power Control}

\section{A. Problem Definition}

The aim of the PCA is to find the powers $P_{i}$ and linear receivers $\mathbf{c}_{i}$, for $i=1, \ldots, K$, such that the total power transmitted by all users is minimized while all user outage constraints are met. Formulating this as an optimization problem, we have

$$
\begin{aligned}
& \min _{\mathbf{P}, \mathbf{C}} \sum_{i=1}^{K} P_{i} \\
& \text { s.t. }\left\{1-\exp \left(\frac{-\sigma^{2}\left(\mathbf{c}_{i}^{\mathrm{T}} \mathbf{c}_{i}\right) \gamma_{i}^{\mathrm{th}}}{G_{i i}\left(\mathbf{c}_{i}^{\mathrm{T}} \mathbf{s}_{i}\right)^{2} P_{i}}\right)\right. \\
&\left.\quad \times \prod_{j \neq i}\left[1+\frac{\gamma_{i}^{\mathrm{th}} G_{i j}\left(\mathbf{c}_{i}^{\mathrm{T}} \mathbf{s}_{j}\right)^{2} P_{j}}{G_{i i}\left(\mathbf{c}_{i}^{\mathrm{T}} \mathbf{s}_{i}\right)^{2} P_{i}}\right]^{-1}\right\} \leq O_{i}^{t}, \\
& P_{i} \geq 0, \mathbf{c}_{i} \in \mathbb{S}^{N} \quad i=1, \ldots, K
\end{aligned}
$$

where we used (5) with the linear receivers included, and $O_{i}^{t}$ are the target outage probabilities. We note that any scalar multiple of the optimal $\mathbf{c}_{i}$ results in the same optimal solution. Thus, to make the problem well defined, we restrict ourselves to $\mathbf{c}_{i} \in \mathbb{R}^{N}$ with $\left\|\mathbf{c}_{i}\right\|=1$, denoted above by the $N$-dimensional unit sphere $\mathbb{S}^{N}$.

We further note that the optimal solution will have outage constraints satisfied with equality. We first observe that outage probability (5) is monotonically increasing in $P_{j}, j \neq i$, and it can be shown to be monotonically decreasing in $P_{i}$. Suppose $\mathbf{P}$ is a power vector where all users have met their outage targets with at least one user $i$ having outage $O_{i}<O_{i}^{t}$. We can then lower $P_{i}$ and still meet all outage constraints. This in turn improves the situation for all other users: they may decrease their own power. Thus, the optimal power vector $\mathbf{P}^{*}$ occurs when $O_{i}=O_{i}^{t}, \forall i$.
The optimization above is equivalent to the following, where the inner optimization has been inserted into the constraint set (see [20] for a similar refinement)

$$
\begin{aligned}
& \min _{\mathbf{P}} \sum_{i=1}^{K} P_{i} \\
& \text { s.t. } \min _{\mathbf{c}_{i} \in \mathbb{S}^{N}}\left\{1-\exp \left(\frac{-\sigma^{2}\left(\mathbf{c}_{i}^{\mathrm{T}} \mathbf{c}_{i}\right) \gamma_{i}^{\mathrm{th}}}{G_{i i}\left(\mathbf{c}_{i}^{\mathrm{T}} \mathbf{s}_{i}\right)^{2} P_{i}}\right)\right. \\
&\left.\times \prod_{j \neq i}\left[1+\frac{\gamma_{i}^{\mathrm{th}} G_{i j}\left(\mathbf{c}_{i}^{\mathrm{T}} \mathbf{s}_{j}\right)^{2} P_{j}}{G_{i i}\left(\mathbf{c}_{i}^{\mathrm{T}} \mathbf{s}_{i}\right)^{2} P_{i}}\right]^{-1}\right\} \leq O_{i}^{t}, \\
& P_{i} \geq 0
\end{aligned}
$$

\section{B. Optimal PCA}

In this section, we describe an iterative algorithm to solve the optimization problem (10).

Taking the logarithm of the outage constraint from (10) and rearranging yields the equivalent constraints

$$
\begin{aligned}
\min _{\mathbf{c}_{i} \in \mathbb{S}^{N}}\left\{\frac{\sigma^{2}\left(\mathbf{c}_{i}^{\mathrm{T}} \mathbf{c}_{i}\right)}{\tilde{G}_{i i}\left(\mathbf{c}_{i}^{\mathrm{T}} \mathbf{s}_{i}\right)^{2} \log \left(\frac{1}{1-O_{i}^{t}}\right)}\right. \\
\left.+\frac{\tilde{G}_{i i}\left(\mathbf{c}_{i}^{\mathrm{T}} \mathbf{s}_{i}\right)^{2} P_{i} \sum_{j \neq i} \log \left(1+\frac{G_{i j}\left(\mathbf{c}_{i}^{\mathrm{T}} \mathbf{s}_{j}\right)^{2} P_{j}}{\tilde{G}_{i i}\left(\mathbf{c}_{i}^{\mathrm{T}} \mathbf{s}_{i}\right)^{2} P_{i}}\right)}{\tilde{G}_{i i}\left(\mathbf{c}_{i}^{\mathrm{T}} \mathbf{s}_{i}\right)^{2} \log \left(\frac{1}{1-O_{i}^{t}}\right)}\right\} \leq P_{i}
\end{aligned}
$$

where $\tilde{G}_{i i}=G_{i i} / \gamma_{i}^{\text {th }}$.

If we view (11) as representing a set of quasi-interference constraints on the power vector $\mathbf{P}$, we can define a new PCA where each user $i$ iteratively attempts to compensate for the interference. At convergence, we would like each of the outage constraints to be met.

With reference to (11), we define

$$
\begin{aligned}
I_{i}\left(\mathbf{P}, \mathbf{c}_{i}\right)= & \frac{\sigma^{2}\left(\mathbf{c}_{i}^{\mathrm{T}} \mathbf{c}_{i}\right)}{\tilde{G}_{i i}\left(\mathbf{c}_{i}^{\mathrm{T}} \mathbf{s}_{i}\right)^{2} \log \left(\frac{1}{1-O_{i}^{t}}\right)} \\
& +\frac{\tilde{G}_{i i}\left(\mathbf{c}_{i}^{\mathrm{T}} \mathbf{s}_{i}\right)^{2} P_{i} \sum_{j \neq i} \log \left(1+\frac{G_{i j}\left(\mathbf{c}_{i}^{\mathrm{T}} \mathbf{s}_{j}\right)^{2} P_{j}}{\tilde{G}_{i i}\left(\mathbf{c}_{i}^{\mathrm{T}} \mathbf{s}_{i}\right)^{2} P_{i}}\right)}{\tilde{G}_{i i}\left(\mathbf{c}_{i}^{\mathrm{T}} \mathbf{s}_{i}\right)^{2} \log \left(\frac{1}{1-O_{i}^{t}}\right)} \\
T_{i}(\mathbf{P})= & \min _{\mathbf{c}_{i}} I_{i}\left(\mathbf{P}, \mathbf{c}_{i}\right)
\end{aligned}
$$

and refer to $I_{i}\left(\mathbf{P}, \mathbf{c}_{i}\right)$ as the interference function to maintain consistency with the framework in [15].

Furthermore, we propose the PCA

$$
\mathbf{P}^{n+1}=\mathbf{T}\left(\mathbf{P}^{n}\right)
$$

where $n$ is the iteration step, $\mathbf{T}(\mathbf{P})=\left[T_{1}(\mathbf{P}), \ldots, T_{K}(\mathbf{P})\right]^{\mathrm{T}}$, and is initialized with powers set to the receiver noise level $P_{i}^{0}=\sigma^{2}$ and MF coefficients $\mathbf{c}_{i}^{0}=\mathbf{s}_{i}, \forall i$. 
This PCA is similar in form to that in [20]; however, we are dealing with outage rather than SIR constraints and average quantities on a slower time scale. In Section V, we will see how a suboptimal variant of the algorithm given by (12) and (13) can be reduced to a new PCA that is similar in form to [20].

Theorem 1 below proposes that (13) is a standard interference function and thus the PCA converges to a fixed solution. The proof is based on the three properties of a standard interference function given in [15] and repeated below.

Definition 1: Interference function $\mathbf{I}(\mathbf{P})$ is standard if, for all $\mathbf{P} \geq 0$, the following properties are satisfied.

1) Positivity- $\mathbf{I}(\mathbf{P})>0$.

2) Monotonicity-If $\mathbf{P} \geq \mathbf{P}^{\prime}$, then $\mathbf{I}(\mathbf{P}) \geq \mathbf{I}\left(\mathbf{P}^{\prime}\right)$.

3) Scalability-For all $\alpha>1, \alpha \mathbf{I}(\mathbf{P})>\mathbf{I}(\alpha \mathbf{P})$.

Theorem 1: $\mathbf{T}(\mathbf{P})$ is a standard interference function.

Proof: We recall that $G_{i j}>0, P_{i}>0$ and $0<O_{i}^{t}<1$. Thus, for any fixed $\mathbf{c}_{i}, I_{i}\left(\mathbf{P}, \mathbf{c}_{i}\right)>0$. Therefore, $T_{i}(\mathbf{P})=$ $\min _{\mathbf{c}_{i}} I_{i}\left(\mathbf{P}, \mathbf{c}_{i}\right)$ is positive and $\mathbf{T}(\mathbf{P})$ is also positive.

To prove monotonicity of (13), we will first show that (12) is monotonic for any fixed $\mathbf{c}_{i}$.

Note that $I_{i}\left(\mathbf{P}, \mathbf{c}_{i}\right)$ is monotonic in all $P_{j}, j \neq i$, thus, we only need to prove monotonicity for $P_{i}$. This is equivalent to proving monotonicity of

$$
y=x \log \left(1+\frac{k}{x}\right)
$$

where $x=\tilde{G}_{i i}\left(\mathbf{c}_{i}^{\mathrm{T}} \mathbf{s}_{i}\right)^{2} P_{i}$ and $k=G_{i j}\left(\mathbf{c}_{i}^{\mathrm{T}} \mathbf{s}_{j}\right)^{2} P_{j}$ is a constant.

It can be shown that (14) is monotonic, provided $k>0$. We have $k=G_{i j}\left(\mathbf{c}_{i}^{\mathrm{T}} \mathbf{s}_{j}\right)^{2} P_{j}$, which is positive, thus (12) is monotonic.

$$
\begin{aligned}
& \text { If } \mathbf{c}_{i}^{*}=\arg \min _{\mathbf{c}_{i}} I_{i}\left(\mathbf{P}, \mathbf{c}_{i}\right) \text {, then, we have } \\
& \qquad \begin{aligned}
T_{i}(\mathbf{P}) & =\min _{\mathbf{c}_{i}} I_{i}\left(\mathbf{P}, \mathbf{c}_{i}\right) \\
& =I_{i}\left(\mathbf{P}, \mathbf{c}_{i}^{*}\right) \\
& \geq I_{i}\left(\mathbf{P}^{\prime}, \mathbf{c}_{i}^{*}\right) \\
& \geq \min _{\mathbf{c}_{i}} I_{i}\left(\mathbf{P}^{\prime}, \mathbf{c}_{i}\right) \\
& =T_{i}\left(\mathbf{P}^{\prime}\right) .
\end{aligned}
\end{aligned}
$$

Thus, $T_{i}(\mathbf{P}) \geq T_{i}\left(\mathbf{P}^{\prime}\right)$ and (13) satisfies the monotonicity property.

To prove scalability, we note that for a fixed $\mathbf{c}_{i}$, we have

$$
\begin{aligned}
I_{i}\left(\alpha \mathbf{P}, \mathbf{c}_{i}\right)= & \frac{\sigma^{2}\left(\mathbf{c}_{i}^{\mathrm{T}} \mathbf{c}_{i}\right)}{\tilde{G}_{i i}\left(\mathbf{c}_{i}^{\mathrm{T}} \mathbf{s}_{i}\right)^{2} \log \left(\frac{1}{1-O_{i}^{t}}\right)} \\
& +\frac{\tilde{G}_{i i}\left(\mathbf{c}_{i}^{\mathrm{T}} \mathbf{s}_{i}\right)^{2} \alpha P_{i} \sum_{j \neq i} \log \left(1+\frac{G_{i j}\left(\mathbf{c}_{i}^{\mathrm{T}} \mathbf{s}_{j}\right)^{2} \alpha P_{j}}{\tilde{G}_{i i}\left(\mathbf{c}_{i}^{\mathrm{T}} \mathbf{s}_{i}\right)^{2} \alpha P_{i}}\right)}{\tilde{G}_{i i}\left(\mathbf{c}_{i}^{\mathrm{T}} \mathbf{s}_{i}\right)^{2} \log \left(\frac{1}{1-O_{i}^{t}}\right)} \\
< & \alpha I_{i}\left(\mathbf{P}, \mathbf{c}_{i}\right)
\end{aligned}
$$

since the $\alpha$ 's cancel in the log term and the noise variance $\sigma^{2}$ is nonzero.
If again $\mathbf{c}_{i}^{*}=\arg \min _{\mathbf{c}_{i}} I_{i}\left(\mathbf{P}, \mathbf{c}_{i}\right)$, then, we have

$$
\begin{aligned}
\alpha T_{i}(\mathbf{P}) & =\min _{\mathbf{c}_{i}} \alpha I_{i}\left(\mathbf{P}, \mathbf{c}_{i}\right) \\
& =\alpha I_{i}\left(\mathbf{P}, \mathbf{c}_{i}^{*}\right) \\
& >I_{i}\left(\alpha \mathbf{P}, \mathbf{c}_{i}^{*}\right) \\
& \geq \min _{\mathbf{c}_{i}} I_{i}\left(\alpha \mathbf{P}, \mathbf{c}_{i}\right) \\
& =T_{i}(\alpha \mathbf{P}) .
\end{aligned}
$$

Thus, $\alpha T_{i}(\mathbf{P})>T_{i}(\alpha \mathbf{P})$, and (13) satisfies the scalability property.

Since $\mathbf{T}(\mathbf{P})$ is a standard interference function, the PCA (13) converges to a final solution $\mathbf{P}^{*}=\mathbf{T}\left(\mathbf{P}^{*}\right)$. This solution is the minimum power required to meet the outage constraints (11). The filter coefficients converge to a new type of multiuser receiver, the MOP receiver. As such, we shall refer to the PCA (13) as the optimal MOP-PCA.

\section{Feasibility}

In the previous section, we implicitly assumed that the constraint set was nonempty. To be more precise, we should say that the fixed-point iteration of (13) converges if and only if there exists a power vector satisfying the outage constraints (10). We have not given conditions for the feasibility of our optimization problem and indeed this appears to be a nontrivial problem. We can however make the following observation: If the set of signature sequences $\left\{\mathbf{s}_{1}, \mathbf{s}_{2}, \ldots, \mathbf{s}_{K}\right\}$ is linearly independent (which can only be true if $K \leq N$ ), then there exists a power vector satisfying the outage constraints (10). To see this, note that under the linear-independence assumption, we could choose $\mathbf{c}_{i}$ to be the decorrelating multiuser detector, in which case $\mathbf{c}_{i}^{\mathrm{T}} \mathbf{s}_{j}=0$ for $j \neq i$, so that the outage for user $i$ is

$$
O_{i}=1-\exp \left(-\frac{\sigma^{2}\left(\mathbf{c}_{i}^{\mathrm{T}} \mathbf{c}_{i}\right) \gamma_{i}^{\mathrm{th}}}{G_{i i}\left(\mathbf{c}_{i}^{\mathrm{T}} \mathbf{s}_{i}\right)^{2} P_{i}}\right)
$$

where $\mathbf{c}_{i}^{\mathrm{T}} \mathbf{s}_{i}>0$ by assumption. Clearly, we can now choose $P_{i}$ so that any outage constraint is satisfied. We can further express the outage probability above as

$$
O_{i}=1-\exp \left(-\frac{1}{C E M_{i}^{\sigma}}\right)
$$

where $\mathrm{CEM}_{i}^{\sigma}$ is the CEM for a single-user situation.

The above linear independence assumption provides a sufficient condition for the existence of a feasible power vector. A full characterization of the feasible (nonempty) constraint sets in terms of the signature sequences, SIR and outage targets, remains an open problem.

\section{POWER Control With SIR Constraints}

In this section, we introduce a power-control problem that considers average SIR constraints rather than dealing directly with outage probability.

To meet our outage-probability constraints, we require that $O_{i} \leq O_{i}^{t}$ for all $i$. Combining this inequality with the upper 
bound in (9), we can define a new constraint on $\mathrm{CEM}_{i}^{\sigma}$, which, when met, will guarantee that our original outage constraints are also met

$$
O_{i} \leq 1-\exp \left(-\frac{1}{C E M_{i}^{\sigma}}\right) \leq O_{i}^{t}, \quad i=1, \ldots, K
$$

Rearranging the right-hand side of (15) yields

$$
\begin{aligned}
\exp \left(-\frac{1}{C E M_{i}^{\sigma}}\right) & \geq 1-O_{i}^{t} \\
C E M_{i}^{\sigma} & \geq \frac{1}{\log \left(\frac{1}{1-O_{i}^{t}}\right)} \\
\overline{S I R}_{i} & \geq \frac{\gamma_{i}^{\mathrm{th}}}{\log \left(\frac{1}{1-O_{i}^{t}}\right)} \\
& =\Gamma_{i}^{\mathrm{th}}
\end{aligned}
$$

where we have used the definition of $\mathrm{CEM}_{i}^{\sigma}$ from (7) and defined a new quantity

$$
\Gamma_{i}^{\mathrm{th}}=\frac{\gamma_{i}^{\mathrm{th}}}{\log \left(\frac{1}{1-O_{i}^{t}}\right)}
$$

called the outage-mapped average SIR threshold. We can now define a new problem

$$
\begin{aligned}
& \min _{\mathbf{P}, \mathbf{C}} \sum_{i=1}^{K} P_{i} \\
& \text { s.t. } P_{i} \geq \frac{\Gamma_{i}^{\mathrm{th}}}{G_{i i}} \frac{\sum_{j \neq i} G_{i j}\left(\mathbf{c}_{i}^{\mathrm{T}} \mathbf{s}_{j}\right)^{2} P_{j}+\sigma^{2}\left(\mathbf{c}_{i}^{\mathrm{T}} \mathbf{c}_{i}\right)}{\left(\mathbf{c}_{i}^{\mathrm{T}} \mathbf{s}_{i}\right)^{2}} \quad i=1, \ldots, K \\
& \qquad P_{i} \geq 0, \quad \mathbf{c}_{i} \in \mathbb{S}^{N} \quad i=1, K
\end{aligned}
$$

where we have rearranged (6) to form an SIR constraint. This problem is mathematically equivalent to the powercontrol problem in [20]; however, we consider average channel gains and the outage-mapped average SIR threshold $\Gamma_{i}^{\text {th }}$ as parameters.

In a similar fashion to [20], the problem is equivalent to

$$
\begin{aligned}
& \min _{\mathbf{P}} \sum_{i=1}^{K} P_{i} \\
& \text { s.t. } P_{i} \geq \frac{\Gamma_{i}^{\mathrm{th}}}{G_{i i}} \min _{\mathbf{c}_{i} \in \mathbb{S}^{N}} \frac{\sum_{j \neq i} G_{i j}\left(\mathbf{c}_{i}^{\mathrm{T}} \mathbf{s}_{j}\right)^{2} P_{j}+\sigma^{2}\left(\mathbf{c}_{i}^{\mathrm{T}} \mathbf{c}_{i}\right)}{\left(\mathbf{c}_{i}^{\mathrm{T}} \mathbf{s}_{i}\right)^{2}} \quad i=1, \ldots, K .
\end{aligned}
$$

\section{A. Suboptimal PCA}

The problem (19) above has an associated PCA given by

$$
\begin{aligned}
J_{i}\left(\mathbf{P}, \mathbf{c}_{i}\right) & =\frac{\Gamma_{i}^{\mathrm{th}}}{G_{i i}} \frac{\sum_{j \neq i} G_{i j}\left(\mathbf{c}_{i}^{\mathrm{T}} \mathbf{s}_{j}\right)^{2} P_{j}+\sigma^{2}\left(\mathbf{c}_{i}^{\mathrm{T}} \mathbf{c}_{i}\right)}{\left(\mathbf{c}_{i}^{\mathrm{T}} \mathbf{s}_{i}\right)^{2}} \\
U_{i}(\mathbf{P}) & =\min _{\mathbf{c}_{i}} J_{i}\left(\mathbf{P}, \mathbf{c}_{i}\right) \\
\mathbf{P}^{n+1} & =\mathbf{U}\left(\mathbf{P}^{n}\right)
\end{aligned}
$$

where $\mathbf{U}(\mathbf{P})=\left[U_{1}(\mathbf{P}), \ldots, U_{K}(\mathbf{P})\right]^{\mathrm{T}}$. We shall refer to this PCA as the outage-mapped MMSE-PCA.

In [20], it was shown that the MMSE filter coefficients $\mathbf{c}_{i}$ minimize (21), and so we have the following iterative algorithm for the above problem

$$
\begin{aligned}
\hat{\mathbf{c}}_{i} & =\operatorname{MMSE}_{i}\left(\mathbf{P}^{n}\right) \\
P_{i}^{n+1} & =\frac{\Gamma_{i}^{\mathrm{th}}}{G_{i i}} \frac{\sum_{j \neq i} P_{j}^{n} G_{i j}\left(\hat{\mathbf{c}}_{i}^{\mathrm{T}} \mathbf{s}_{j}\right)^{2}+\sigma^{2}\left(\hat{\mathbf{c}}_{i}^{\mathrm{T}} \hat{\mathbf{c}}_{i}\right)}{\left(\hat{\mathbf{c}}_{i}^{\mathrm{T}} \mathbf{s}_{i}\right)^{2}}
\end{aligned}
$$

where

$$
\operatorname{MMSE}_{i}(\mathbf{P})=\rho\left(\mathbf{S}_{-i} \mathbf{D}_{-i} \mathbf{S}_{-i}^{\mathrm{T}}+\sigma^{2} \mathbf{I}\right)^{-1} \mathbf{S}_{i}
$$

$\rho$ is a scalar chosen so that $\left\|\operatorname{MMSE}_{i}(\cdot)\right\|=1$; the matrix $\mathbf{S}_{-i}$ is of dimension $N \times(K-1)$ having the same form as $\mathbf{S}$, omitting the $i$ th column; and $\mathbf{D}_{-i}$ is a $(K-1) \times(K-1)$ diagonal matrix with entries $P_{1} G_{11}, \ldots, P_{K} G_{K K}$, omitting $P_{i} G_{i i}$.

The receiver estimate $\hat{\mathbf{c}}_{i}$ is updated on each iteration and the PCA is initialized with powers set to the noise level and MF coefficients. The convergence proof of this PCA mirrors that in [20] and relies on the right-hand side of (21) being a standard interference function.

Since we have used the upper bound on $O_{i}$ in the derivation above, the solution is suboptimal; however, we guarantee the outage constraints from the right-hand side of (15). Recall that in the region of interest, the outage bounds are tight, and so we expect that this PCA will result in near-optimal performance as compared to the optimal MOP-PCA.

\section{HYBRID PCA}

The reduced computational requirements of the MMSE-PCA in the previous section is a key practical advantage, since the minimizer of the standard interference function $J_{i}(\cdot, \cdot)$ is known in closed form. Contrast this to the MOP-PCA of Section IV-B: in practice, it requires complex iterative optimization methods for computation since the minimization is over a nonlinear function of the receiver filter.

Envisage a new algorithm, where we take the MOP-PCA and replace this complex minimization with the closed-form MMSE receiver solution. In [12], it was noted that maximizing $\mathrm{CEM}_{i}$ is often nearly equivalent to minimizing outage probability, since the outage-probability bounds are very tight (their ratio being often near 1). Given that the bounds (9) for $\mathrm{CEM}_{i}^{\sigma}$ have the same form as in [12], we are effectively using the MMSE receiver to reduce outage probability. Obviously, such an algorithm benefits from having greater computational efficiency. Furthermore, no bound is required: we would expect the final outages to be met with equality, in contrast to the MMSE-PCA, where this is not necessarily the case, and depend on the tightness of the bound.

We define a new interference function

$$
H_{i}(\mathbf{P})=I_{i}\left(\mathbf{P}, \operatorname{MMSE}_{i}(\mathbf{P})\right)
$$

where $I_{i}\left(\mathbf{P}, \mathbf{c}_{i}\right)$ was previously defined in (12). 
The new PCA is then of the form

$$
\mathbf{P}^{n+1}=\mathbf{H}\left(\mathbf{P}^{n}\right)
$$

where again, $n$ denotes the iteration step, $\mathbf{H}(\mathbf{P})=\left[H_{1}(\mathbf{P})\right.$, $\left.\ldots, H_{K}(\mathbf{P})\right]^{\mathrm{T}}$, and the algorithm is initialized with powers set to the receiver noise level and the MF coefficients. We shall refer to this algorithm as the hybrid outage-probability (HOP) PCA.

We conjecture that if the MMSE-PCA converges, indeed doing so whenever (19) is feasible, then the HOP-PCA will converge to a solution $\mathbf{P}^{*}$ that is less than the fixed-point solution of the MMSE-PCA. This conjecture is supported by simulation. Furthermore, the following theorem proposes that, by starting from a common initial power vector, the $n$th iteration generated by the HOP-PCA is bounded above and below by the $n$th iteration vectors generated by the MMSE- and MOP-PCAs, respectively. We conclude that if the fixed points of the MOPand MMSE-PCAs are very close, as determined by the tight bound on outage, then, for all practical engineering purposes, the HOP-PCA will, at the very least, converge to some small region of powers above the optimal fixed-point solution.

Lemma 1: For any fixed power vector $\mathbf{P}, T_{i}(\mathbf{P}) \leq H_{i}(\mathbf{P}) \leq$ $U_{i}(\mathbf{P})$.

Proof: We can rewrite the left-hand-side inequality as

$$
\min _{\mathbf{c}_{i}} I_{i}\left(\mathbf{P}, \mathbf{c}_{i}\right) \leq I_{i}\left(\mathbf{P}, \operatorname{MMSE}_{i}(\mathbf{P})\right)
$$

We can also rewrite the right-hand side inequality as

$$
I_{i}\left(\mathbf{P}, \operatorname{MMSE}_{i}(\mathbf{P})\right) \leq J_{i}\left(\mathbf{P}, \operatorname{MMSE}_{i}(\mathbf{P})\right)
$$

where

$$
J_{i}\left(\mathbf{P}, \mathbf{c}_{i}\right)=\frac{\sigma^{2}\left(\mathbf{c}_{i}^{\mathrm{T}} \mathbf{c}_{i}\right)+\sum_{j \neq i} G_{i j}\left(\mathbf{c}_{i}^{\mathrm{T}} \mathbf{s}_{j}\right)^{2} P_{j}}{\tilde{G}_{i i}\left(\mathbf{c}_{i}^{\mathrm{T}} \mathbf{s}_{i}\right)^{2} \log \left(\frac{1}{1-O_{i}^{t}}\right)}
$$

is the interference function for the MMSE-PCA (20) put into a form similar to (12). To show that this inequality holds, we note that for each $i \neq j$, the summation term in (12) can be bounded from above as follows

$$
\begin{gathered}
\tilde{G}_{i i}\left(\mathbf{c}_{i}^{\mathrm{T}} \mathbf{s}_{i}\right)^{2} P_{i} \log \left(1+\frac{G_{i j}\left(\mathbf{c}_{i}^{\mathrm{T}} \mathbf{s}_{j}\right)^{2} P_{j}}{\tilde{G}_{i i}\left(\mathbf{c}_{i}^{\mathrm{T}} \mathbf{s}_{i}\right)^{2} P_{i}}\right) \\
\leq \tilde{G}_{i i}\left(\mathbf{c}_{i}^{\mathrm{T}} \mathbf{s}_{i}\right)^{2} P_{i} \frac{G_{i j}\left(\mathbf{c}_{i}^{\mathrm{T}} \mathbf{s}_{j}\right)^{2} P_{j}}{\tilde{G}_{i i}\left(\mathbf{c}_{i}^{\mathrm{T}} \mathbf{s}_{i}\right)^{2} P_{i}} \\
\quad=G_{i j}\left(\mathbf{c}_{i}^{\mathrm{T}} \mathbf{s}_{j}\right)^{2} P_{j}
\end{gathered}
$$

since $\log (1+x) \leq x$, provided $x \geq 0$. This bound is exactly the term appearing within the summation in (28).

Lemma 2: Given any three power vectors satisfying ${ }^{T} \mathbf{P} \leq$ ${ }^{H} \mathbf{P} \leq{ }^{U} \mathbf{P}$, then $T_{i}\left({ }^{T} \mathbf{P}\right) \leq H_{i}\left({ }^{H} \mathbf{P}\right) \leq U_{i}\left({ }^{U} \mathbf{P}\right)$.
Proof: Immediate from Lemma 1 and the monotonicity properties of $T_{i}(\cdot)$ and $U_{i}(\cdot)$.

Let

$$
\begin{aligned}
{ }^{T} \mathbf{P}^{n+1} & =\mathbf{T}\left({ }^{T} \mathbf{P}^{n}\right) \\
{ }^{H} \mathbf{P}^{n+1} & =\mathbf{H}\left({ }^{H} \mathbf{P}^{n}\right) \\
{ }^{U} \mathbf{P}^{n+1} & =\mathbf{U}\left({ }^{U} \mathbf{P}^{n}\right)
\end{aligned}
$$

with

$$
{ }^{T} \mathbf{P}^{0}={ }^{H} \mathbf{P}^{0}={ }^{U} \mathbf{P}^{0}=\mathbf{P}^{0} .
$$

Theorem 2: For all iterations $n$

$$
{ }^{T} \mathbf{P}^{n+1} \leq{ }^{H} \mathbf{P}^{n+1} \leq{ }^{U} \mathbf{P}^{n+1} .
$$

Proof: Immediate from Lemma 1 and Lemma 2.

\section{LARGE-SYSTEM ANALYSIS (LSA)}

The MMSE filter coefficients $\mathbf{c}_{i}$ minimize the interference function (21) in Section V-A above. Equivalently, $\mathbf{c}_{i}$ maximizes the average SIR of user $i$. The corresponding maximal $\overline{\text { SIR }}$ for user $i$ is given by

$$
\overline{S I R}_{i}=P_{i} G_{i i} \mathbf{s}_{i}^{\mathrm{T}}\left(\mathbf{S}_{-i} \mathbf{D}_{-i} \mathbf{S}_{-i}^{\mathrm{T}}+\sigma^{2} \mathbf{I}\right)^{-1} \mathbf{s}_{i}
$$

which we can use to restate the optimization problem (19) of Section V as follows:

$$
\begin{aligned}
& \min _{\mathbf{P}} \sum_{i=1}^{K} P_{i} \\
& \text { s.t. } \overline{S I R}_{i} \geq \Gamma_{i}^{\text {th }} \\
& \quad P_{i} \geq 0 \quad i=1, \ldots, K .
\end{aligned}
$$

Recall that the optimal solution to the optimization problems of Section IV-A occurs when all users meet their outage constraints with equality. A similar argument can be constructed for this problem, dealing with outage-mapped average SIR constraints. We then reduce this problem to one where we solve the system of equations

$$
P_{i} G_{i i} \mathbf{s}_{i}^{\mathrm{T}}\left(\mathbf{S}_{-i} \mathbf{D}_{-i} \mathbf{S}_{-i}^{\mathrm{T}}+\sigma^{2} \mathbf{I}\right)^{-1} \mathbf{s}_{i}=\Gamma_{i}^{\mathrm{th}}
$$

for all $i=1, \ldots, K$.

The LSA in [19] derives a closed-form expression that the left-hand side of (30) converges to, in probability, as $N$, $K \rightarrow \infty$ and the number of users per degree of freedom, or system loading, $\alpha \equiv K / N$ remains fixed. This SIR, in the limit, is a deterministic quantity for each user $i$, denoted $\beta_{i}^{*}$. Somewhat surprisingly, the result enables the uncoupling of each user in the SIR expression above, by representing each user with an effective interference. Using this decoupled result, we can then solve a new system of equations that, in a large system, is equivalent to (30), and where each individual equation can be solved independently of each other, resulting in a decentralized and noniterative PCA. 
In a finite-sized system with MMSE receivers, the LSA result allows us to approximate the average SIR for a given user $i$, denoted $\beta_{i}$. The average SIR approximation is given by

$$
\beta_{i} \approx \frac{P_{i} G_{i i}}{\sigma^{2}+\frac{1}{N} \sum_{j=1}^{K} I\left(P_{j} G_{j j}, P_{i} G_{i i}, \beta_{i}\right)}
$$

where $I\left(P_{j} G_{j j}, P_{i} G_{i i}, \beta_{i}\right)$ is the effective interference term contributed from the $j$ th user, and is defined as

$$
I\left(P_{j} G_{j j}, P_{i} G_{i i}, \beta_{i}\right) \equiv \frac{P_{j} G_{j j} P_{i} G_{i i}}{P_{i} G_{i i}+P_{j} G_{j j} \beta_{i}} .
$$

Combining (31) and (32) yields

$$
\beta_{i} \approx \frac{P_{i} G_{i i}}{\sigma^{2}+\frac{1}{N} \sum_{j=1}^{K} \frac{P_{j} G_{j j} P_{i} G_{i i}}{P_{i} G_{i i}+P_{j} G_{j j} \beta_{i}} .}
$$

For the remaining analysis, we shall assume (33) holds with equality.

Let $\alpha_{i}^{*}=\beta_{i} / P_{i} G_{i i}$. Equation (33) becomes

$$
\alpha_{i}^{*} P_{i} G_{i i}=\frac{P_{i} G_{i i}}{\sigma^{2}+\frac{1}{N} \sum_{j=1}^{K} \frac{P_{j} G_{j j}}{1+P_{j} G_{j j} \alpha_{i}^{*}}}
$$

or

$$
\alpha^{*}=\frac{1}{\sigma^{2}+\frac{1}{N} \sum_{j=1}^{K} \frac{P_{j} G_{j j}}{1+P_{j} G_{j j} \alpha *}}
$$

since $\alpha_{i}^{*}$ does not depend on $i$.

Substituting $P_{j} G_{j}=\beta_{j} / \alpha^{*}=\Gamma_{j}^{\mathrm{th}} / \alpha^{*}$ into (34) gives

$$
\begin{aligned}
\alpha^{*} & =\frac{1}{\sigma^{2}+\frac{1}{N} \sum_{j=1}^{K} \frac{\frac{\beta_{j}}{\alpha^{*}}}{1+\frac{\beta_{j}}{\alpha^{*}} \alpha^{*}}} \\
& =\frac{1}{\sigma^{2}+\frac{1}{\alpha^{*}} \frac{1}{N} \sum_{j=1}^{K} \frac{\Gamma_{j}^{\mathrm{th}}}{1+\Gamma_{j}^{\mathrm{th}}}} .
\end{aligned}
$$

The nontrivial solution to the fixed point (35) is given by

$$
\alpha^{*}=\frac{1-\frac{1}{N} \sum_{j=1}^{K} \frac{\Gamma_{j}^{\text {th }}}{1+\Gamma_{j}^{\text {th }}}}{\sigma^{2}}=\frac{\Gamma_{i}^{\text {th }}}{P_{i} G_{i i}}
$$

where again, $P_{i} G_{i i}=\Gamma_{i}^{\mathrm{th}} / \alpha^{*}$. Rearranging the left-hand side for $P_{i}$ gives the power solution of the $i$ th user

$$
P_{i}=\frac{\Gamma_{i}^{\mathrm{th}}}{G_{i i}} \frac{\sigma^{2}}{1-\frac{1}{N} \sum_{j=1}^{K} \frac{\Gamma_{j}^{\mathrm{th}}}{1+\Gamma_{j}^{\mathrm{th}}}}
$$

where we have the necessary and sufficient condition

$$
\frac{1}{N} \sum_{j=1}^{K} \frac{\Gamma_{j}^{\mathrm{th}}}{1+\Gamma_{j}^{\mathrm{th}}}<1
$$

for a solution to exist. This solution is a generalization of a previous result given in [19] for a finite set of $J$ user classes. Here, we consider a limiting regime of $K \rightarrow \infty$ classes of users.

The expression (37) allows for a totally decentralized and noniterative PCA since we have a closed-form expression that only requires a user's channel gain and a common term comprising all outage-mapped $\overline{\mathrm{SIR}}$ thresholds. In practice, such a decentralized implementation would involve two stages. In the first stage, the left-hand side of (38) would need to be broadcast to all users. Here we make the reasonable assumptions that the individual outage-mapped threshold $\Gamma_{i}^{\text {th }}$ and receiver noise power $\sigma^{2}$ are known by each user, while the uplink channel gain $G_{i i}$ can be estimated from the respective downlink channel gain. Having all required parameters for (37), each user can then compute their required transmit power. The second stage involves computation of all user transmit powers at the BSR to form the MMSE expression, giving the required linear receivers for each user.

We shall refer to this PCA as the LSA-PCA.

\section{Simulation Results}

Our simulations consider a single circular CDMA cell with radius $1 \mathrm{~km}$. We assume a uniform distribution on the location of the users within the cell who are each subject to a distancedependent loss (loss exponent 4) and lognormal (zero mean, $8 \mathrm{~dB}$ variance) shadowing. Unless otherwise stated, a processing gain of 32 was chosen, corresponding to a chip rate of $1.2288 \mathrm{MHz}$ and an encoder input rate of $38.4 \mathrm{~kb} / \mathrm{s}$ under the cdma2000 specification [23]. Furthermore, an AWGN noise power equal to $\sigma^{2}=10^{-13}$ was chosen, corresponding to approximately a $1 \mathrm{MHz}$ bandwidth.

We define three user classes, each having typical outage probability and SIR threshold pairs as $\{(5 \%, 10 \mathrm{~dB})$, $(10 \%, 8 \mathrm{~dB}),(20 \%, 6 \mathrm{~dB})\}$. We assign $25 \%$ of users to the first class, $50 \%$ to the second, and the remaining to the third.

In all simulations that follow, user signature sequences are chosen randomly, initial filter coefficients are set to the MF, and initial user powers are set to the receiver noise power $\sigma^{2}=10^{-13}$ where appropriate.

\section{A. Optimal, Outage-Mapped, and Hybrid PCAs}

In this section, we compare the optimal MOP-PCA, outagemapped MMSE-PCA, and the hybrid HOP-PCA. The MOPPCA is computed with (12) and (13), where we employ sequential quadratic programming [24] to minimize the interference function in (13). The MOP-PCA is computed from (26) and (27) once we have obtained the outage-mapped average SIR thresholds (18) from each user's outage-probability target. Finally, the HOP-PCA is computed from (26) and (27). We declare convergence of an algorithm once successive iterations yield an average per-user power of within $1 \%$.

For $K=4,8,16,32$ users, Fig. 1 shows in log scale the sum power of all users as a function of the iteration step. All three algorithms yield almost indistinguishable results, verifying earlier claims on the tightness of the outage-probability bounds used in the MMSE-PCA.

Fig. 2 considers a system of $K=32$ users. It shows the convergence of the outage probabilities to their targets, for each 


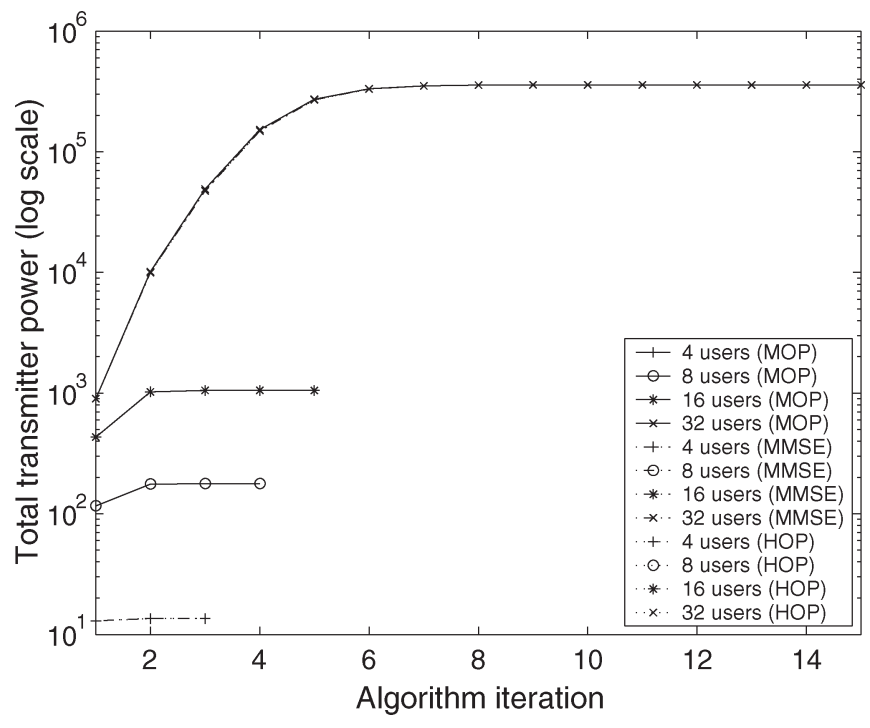

Fig. 1. Total transmitter power with $4-32$ users.

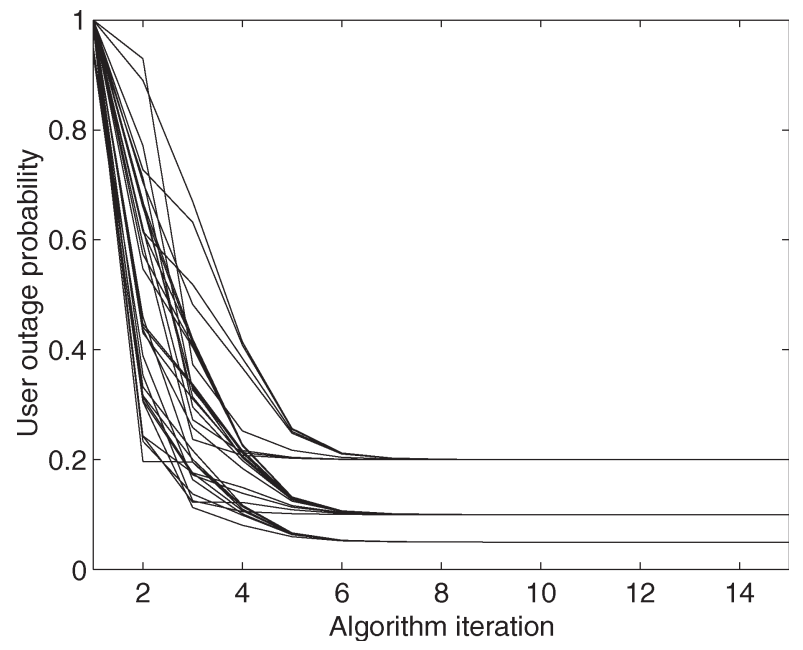

Fig. 2. Outage probability for the MOP-PCA with 32 users.

user, as a function of the iteration step for the MOP-PCA. We clearly see the three outage-probability classes $(5 \%, 10 \%$, and $20 \%$ ) at convergence.

\section{B. Performance Comparison: Variable Versus Fixed Linear Filters}

This section compares the performance of the developed algorithms, where we have a choice over linear filters, to a similar one where they remain fixed from the outset. We consider (24), where the linear filters are fixed to the MF $\hat{\mathbf{c}}_{i}=\mathbf{c}_{i}=\mathbf{s}_{i}$, and refer to this variant as the MF-PCA. For brevity, we concentrate on the MMSE-PCA in our comparison, given the observation in the previous section, where the MOP- and HOP-PCAs have yielded almost indistinguishable results.

We maintain the specified QoS classes, again mapping them to an associated average SIR constraint using (18). We consider 1000 independent simulation runs for each algorithm. Signature sequences are chosen independently between runs; however, sequences are common across simulations for each algorithm and specific value of $K$.

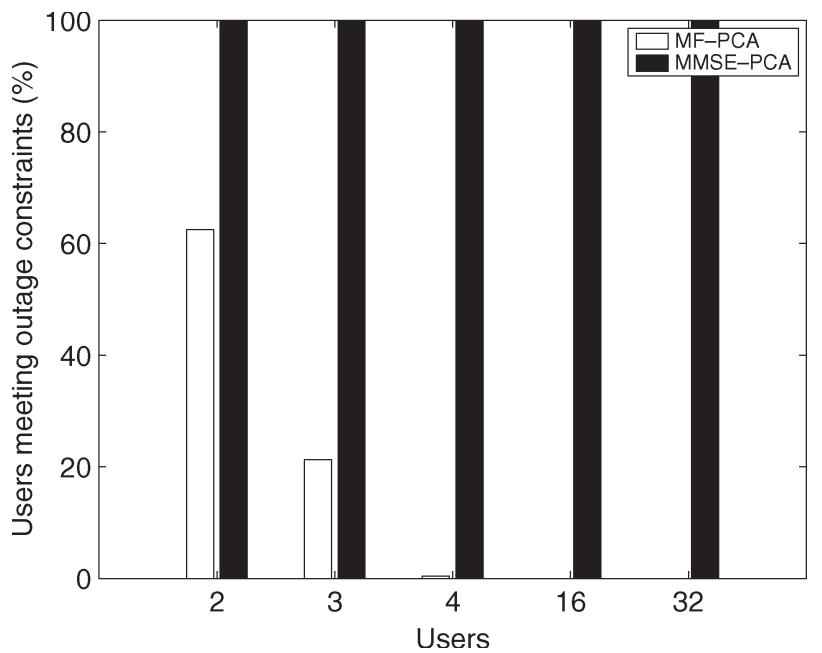

Fig. 3. Total transmitter power with $2-32$ users, averaged over 1000 simulations.

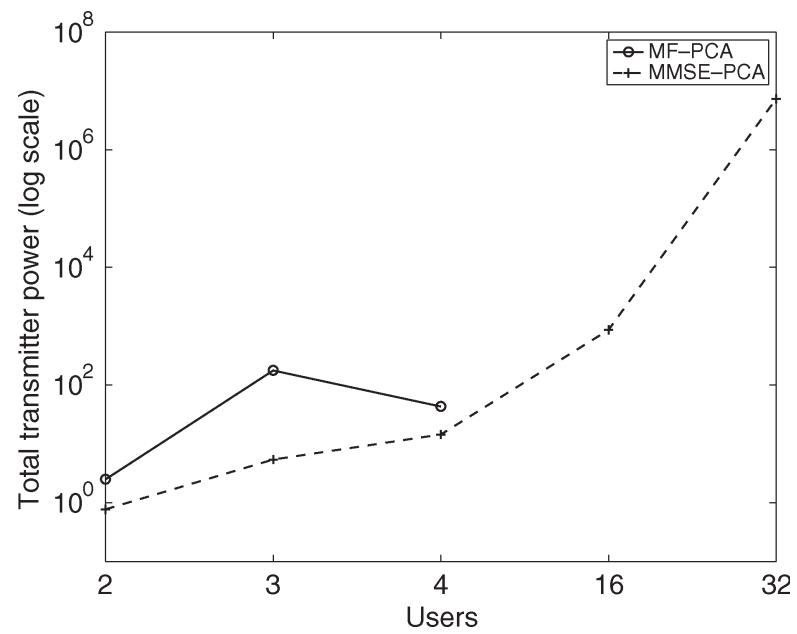

Fig. 4. Percentage of users meeting constraints, averaged over 1000 simulations.

For $K=2,3,4,16,32$ users, Fig. 3 shows the total power allocation, averaged over each simulation run. Simulations where the problem was infeasible are excluded from this figure and is the reason for the MF-PCA plot stopping at $K=4$. This result clearly demonstrates that a performance advantage can be achieved through a joint optimization over user transmit power and linear receivers.

Note the reduction in total power for the MF-PCA as $K$ was increased from three to four users. This can be attributed to the low number of feasible runs $(0.425 \%)$ in the scenario for $K=4$ : only those runs where users had "good" signature sequences would result in a solution where all constraints had been met. In the scenarios where $K=3$, there was less competition among users (as each had a higher degree of freedom) and a greater number of less-efficient signature-sequence combinations were possible, in the sense that more feasible runs were possible at the expense of a higher total power on average.

To appreciate the relative difficulty of obtaining a feasible run, Fig. 4 shows the percentage of users that met their outage constraints, averaged over all simulation runs. 


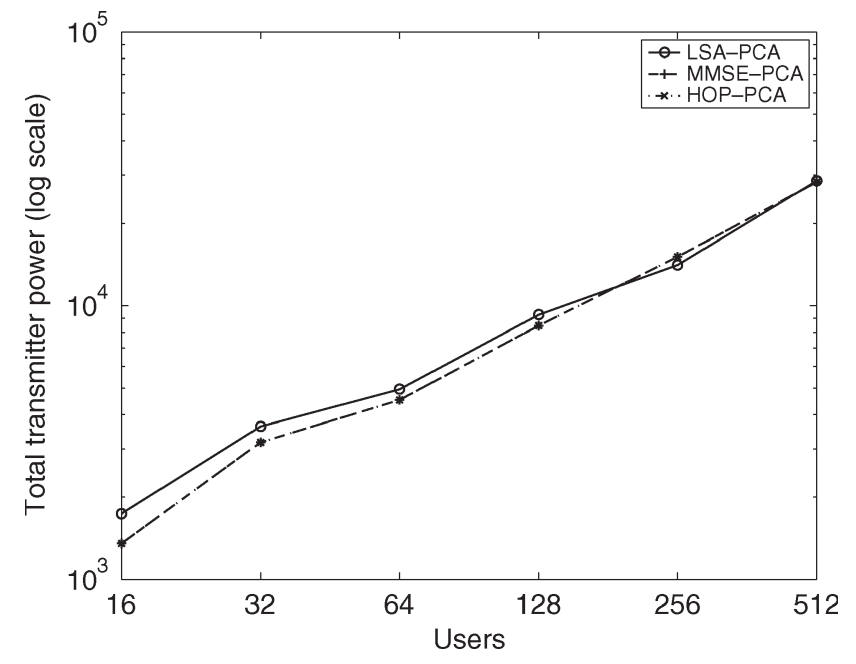

Fig. 5. Comparison between the LSA-, MMSE-, and HOP-PCAs with $K=$ $16, \ldots, 512$ users and $\alpha=0.75$.

\section{Large Systems}

We now consider the large-system results for $K=16,32,64$, $128,256,512$ users and compare the LSA-PCA with both of the HOP- and MMSE-PCAs. A fixed system loading of $\alpha=0.75$ was chosen, with other parameters as above.

Fig. 5 shows in $\log$ scale the sum power of all users in each simulation scenario. As the system size is scaled upwards, the accuracy of the LSA-PCA improves significantly until it becomes indistinguishable from the HOP- and MMSEPCAs. For medium-sized systems, the approximate result gives excellent performance, considering the reduced computational complexity and decentralized nature of the result.

Fig. 6 shows the percentage of users that did not meet their outage constraints in each simulation scenario with the LSAPCA implementation. Similar statistics are not shown for the HOP- and MMSE-PCAs, as all users in all scenarios met their outage constraints with equality. Given the closeness of the results observed in Fig. 5, we further quantify the average deviation between the outage-probability specification and simulated outage probability in Fig. 6, for each user not meeting their outage target with the LSA-PCA. We see that under all scenarios, this deviation is small-ranging from $3 \%$ to under $1 \%$ - and improves as the size of the system is scaled upwards. This statistic is important as it validates the LSA method, even for smaller sized systems.

\section{CONCLUSION}

This paper introduced a new power-control problem that aims to jointly optimize user transmit powers and linear receiver filters according to individual user outage-probability specifications. An iterative algorithm to solve this problem optimally was developed and convergence proved. A bound on outage probability enabled a mapping to take place between outage and an average SIR threshold. From this, a suboptimal PCA-MUD that utilized the well-known MMSE receiver was developed. The approximation to the optimal solution was found to be

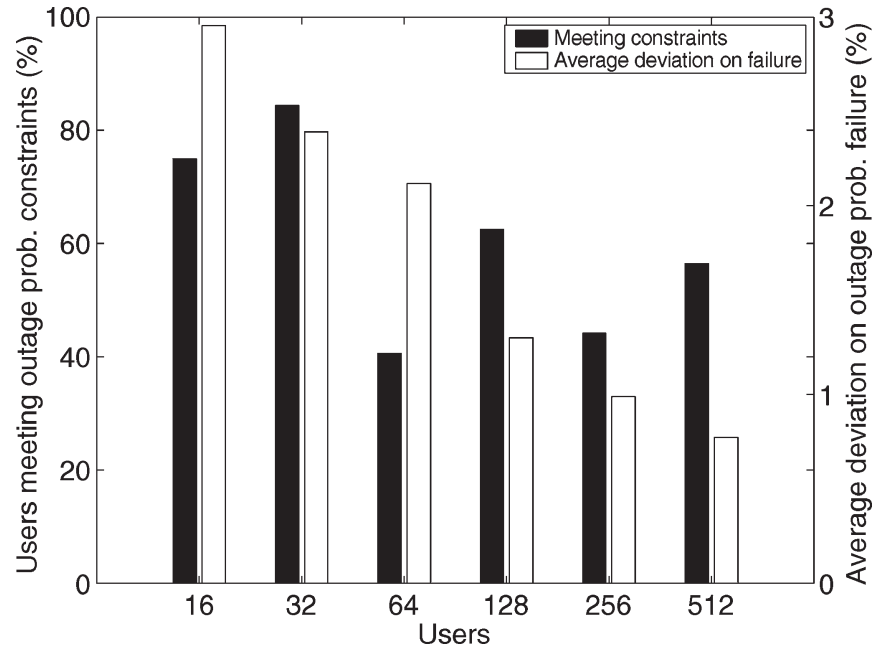

Fig. 6. Performance of the LSA-PCA in meeting user outage-probability constraints, and the average deviation between resultant user outage probability and respective targets for those users with an inadequate QoS.

exceptionally close in the region of interest. A further suboptimal PCA-MUD was introduced, which combined the benefits of the previous two: high computational efficiency with a result that is closer to the optimal.

Large-system approximations were used to decouple complex SIR expressions when the number of users and degrees of freedom approach infinity, with a fixed ratio between the two. This approximation enabled a closed-form solution to an outage-mapped, joint power control, and MMSE multiuser receiver optimization problem. In a finite-sized system, the approximate solution was found to be close to the full HOPand MMSE-PCA solutions, giving excellent results for medium to large finite-sized systems.

Finally, we note that there are a number of important open issues that arise from the results presented in this paper.

1) The extension of the algorithms to more general fastfading distributions such as Rician and Nakagami- $m$. Some initial work in this direction is presented in [25].

2) Investigation of feasibility conditions for the outageconstrained power-control problems. As an example, one may be interested in controlling the admission of users to the network so that the resultant outage constraints are feasible with high probability (see [26] for example).

3) Examination of the extent to which the algorithms can be decentralized and based on noisy local measurements. The challenge here is to develop stochastic PCAs along the lines of those in [13] and [20], which are applicable for systems with outage constraints.

These issues are currently under investigation.

\section{REFERENCES}

[1] J. Aein, "Power balancing in system employing frequency reuse," COMSAT Tech. Rev., vol. 3, no. 2, pp. 277-300, 1973.

[2] R. Nettleton and H. Alavi, "Power control for a spread spectrum radio system," in IEEE Vehicular Technology Conf., Toronto, ON, Canada, 1983, pp. 242-246. 
[3] J. Zander, "Performance of optimum transmitter power control in cellular radio systems," IEEE Trans. Veh. Technol., vol. 41, no. 1, pp. 57-62, Feb. 1992.

[4] S. A. Grandhi, R. Vijayan, D. J. Goodman, and J. Zander, "Centralized power control in cellular radio systems," IEEE Trans. Veh. Technol., vol. 42, no. 4, pp. 466-468, Nov. 1993.

[5] D. Mitra, "An asynchronous distributed algorithm for power control in cellular radio systems," in 4th WINLAB Workshop 3rd Generation Wireless Information Networks, East Brunswick, NJ, 1993, pp. 249-259.

[6] G. J. Foschini and Z. Miljanic, "A simple distributed autonomous power control algorithm and its convergence," IEEE Trans. Veh. Technol., vol. 42, no. 4, pp. 541-646, Nov. 1993.

[7] S. Grandhi, J. Zander, and R. Yates, "Constrained power control," Int. J. Wireless Pers. Commun., vol. 1, no. 4, pp. 257-270, 1995.

[8] S. V. Hanly, "An algorithm of combined cell-site selection and power control to maximize cellular spread spectrum capacity," IEEE J. Sel. Areas Commun., vol. 13, no. 7, pp. 1332-1340, Sep. 1995.

[9] R. D. Yates and C. Y. Huang, "Integrated power control and base station assignment," IEEE Trans. Veh. Technol., vol. 44, no. 3, pp. 638-644, Aug. 1995.

[10] J. Zander, "Transmitter power control for co-channel interference management in cellular radio systems," in 4th WINLAB Workshop on 3rd Generation Wireless Information Networks, New Brunswick, NJ, 1993, pp. 1281-1285.

[11] D. Julian, M. Chiang, and D. O'Neill, "Robust and QoS constrained optimization of power control in wireless cellular networks," in IEEE Vehicular Technology Conf., Atlantic City, NJ, 2001, vol. 3, pp. 1932-1936.

[12] S. Kandukuri and S. Boyd, "Optimal power control in interference-limited fading wireless channels with outage-probability specifications," IEEE Trans. Wireless Commun., vol. 1, no. 1, pp. 46-55, Jan. 2002.

[13] S. Ulukus and R. D. Yates, "Stochastic power control for cellular radio systems," IEEE Trans. Commun., vol. 46, no. 6, pp. 784-798, Jun. 1998.

[14] N. Bui and S. Dey, "Optimal power control in CDMA over Markov fading channels," in Proc. IEEE Int. Symp. Information Theory, Lausanne, Switzerland, Jul. 2002, p. 79.

[15] R. D. Yates, "A framework for uplink power control in cellular radio systems," IEEE J. Sel. Areas Commun., vol. 13, no. 7, pp. 1341-1347, Sep. 1995

[16] S. Verdú, Multiuser Detection. Cambridge, NY: Cambridge Univ. Press, 1998.

[17] _ , "Minimum probability of error for asynchronous Gaussian multiple-access channels," IEEE Trans. Inf. Theory, vol. IT-32, no. 1, pp. 85-96, Jan. 1986

[18] U. Madhow and M. L. Honig, "MMSE interference suppression for directsequence spread-spectrum CDMA," IEEE Trans. Commun., vol. 42, no. 12, pp. 3178-3188, Dec. 1994.

[19] D. N. C. Tse and S. V. Hanly, "Linear multiuser receivers: Effective interference, effective bandwidth and user capacity," IEEE Trans. Inf. Theory, vol. 45, no. 2, pp. 641-657, Mar. 1999.

[20] S. Ulukus and R. D. Yates, "Adaptive power control and MMSE interference suppression," Wireless Netw., Special Issue on Multiuser Detection in Wireless Communications, vol. 4, no. 6, pp. 489-496, 1997.

[21] A. F. Almutairi, S. L. Miller, H. A. Latchman, and T. F. Wong, "Power control algorithm for MMSE receiver based CDMA systems," IEEE Commun. Lett., vol. 4, no. 11, pp. 346-348, Nov. 2000.

[22] A. Yener, R. D. Yates, and S. Ulukus, "Interference management for CDMA systems through power control, multiuser detection, and beamforming," IEEE Trans. Commun., vol. 49, no. 7, pp. 1227-1239, Jul. 2001.

[23] Working document towards submission of RTT candidate to ITU-R IMT-2000 processed 'The cdma2000 RTT candidate submission', ITU-R Draft Doc. TR45.5, Jun. 1998.

[24] R. Fletcher, Practical Methods of Optimization. New York: Wiley, 1980.

[25] J. Papandriopoulos, J. Evans, and S. Dey, "Outage-based power control for generalized multiuser fading channels," in IEEE Int. Conf. Communications, Paris, France, Jun. 2004, vol. 1, pp. 327-331.

[26] J. S. Evans and D. Everitt, "Effective bandwidth based admission control for multi-service CDMA cellular networks," IEEE Trans. Veh. Technol., vol. 48, no. 1, pp. 36-46, Jan. 1999.

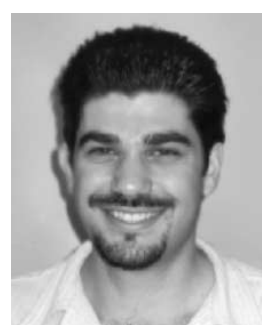

John Papandriopoulos ( $\left.\mathrm{S}^{\prime} 01\right)$ was born in Melbourne, Australia, in 1978. He received the combined B.E. degree in communications engineering and the B.App.Sci. degree in computer science from the Royal Melbourne Institute of Technology (RMIT University), in 2001. Since March 2002, he has been pursuing the Ph.D. degree with the Department of Electrical and Electronic Engineering, at the University of Melbourne, Australia.

His research interests are in code division multiple access (CDMA) signal processing, cross-layer optimization of wireless communication networks, and distributed systems. He enjoys delving into practical engineering implementations, and has spent some time with Telstra, Agilent Technologies, and the 3G Mobile R\&D Division of NEC Australia.

Mr. Papandriopoulos received the J. N. McNicol Prize (University Medal) upon graduating from RMIT University in 2002. Since 2003, he has served as the Chairperson of the University of Melbourne IEEE Student Branch.

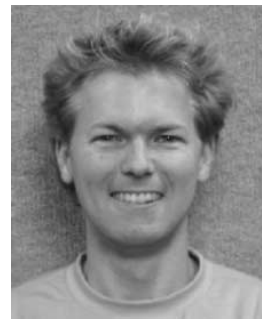

Jamie Evans (S'93-M'98) was born in Newcastle, Australia, in 1970. He received the B.S. degree in physics and the B.E. degree in computer engineering from the University of Newcastle, Callaghan, in 1992 and 1993, respectively, where he received the University Medal upon graduation. He received the M.S. and the Ph.D. degrees from the University of Melbourne, Parkville, Australia, in 1996 and 1998, respectively, both in electrical engineering, and was awarded the Chancellor's Prize for excellence for his $\mathrm{Ph} . \mathrm{D}$. thesis.

From March 1998 to June 1999, he was a Visiting Researcher in the Department of Electrical Engineering and Computer Science, University of California, Berkeley. He returned to Australia to take up a position as Lecturer at the University of Sydney, Sydney, Australia, where he stayed until July 2001. Since that time, he has been with the Department of Electrical and Electronic Engineering, University of Melbourne, where he is now an Associate Professor. His research interests are in communications theory, information theory, and statistical signal processing with current focus on wireless communications networks.

Dr. Evans currently serves on the Editorial Board of the IEEE TRANSACTIONS ON WIRELESS COMMUNICATIONS

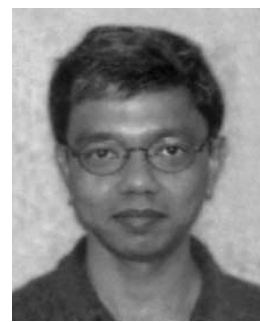

Subhrakanti Dey (S'94-M'96) was born in Calcutta, India, in 1968. He received the B.Tech. and M.Tech. degrees from the Department of Electronics and Electrical Communication Engineering, Indian Institute of Technology, Kharagpur, India, in 1991 and 1993, respectively, and the Ph.D. degree from the Department of Systems Engineering, Research School of Information Sciences and Engineering, Australian National University, Canberra, Australia, in 1996.

He has been with the Department of Electrical and Electronic Engineering, University of Melbourne, Parkville, Australia, since February 2000, first as a Senior Lecturer, and then as an Associate Professor. From September 1995 to September 1997, and September 1998 to February 2000, he was a Postdoctoral Research Fellow with the Department of Systems Engineering, Australian National University. From September 1997 to September 1998, he was a Postdoctoral Research Associate with the Institute for Systems Research, University of Maryland, College Park. His current research interests include signal processing for telecommunications, wireless communications, and networks, performance analysis of communication networks, stochastic and adaptive estimation and control, and statistical and adaptive signal processing.

Dr. Dey currently serves on the Editorial Board of the IEEE TRANSACTIONS on AutOMATic ConTrol and Elsevier Systems and Control Letters. 